\title{
A combination of omental flap and growth factor therapy induces arteriogenesis and increases myocardial perfusion in chronic myocardial ischemia: Evolving concept of biologic coronary artery bypass grafting
}

Kiyoaki Takaba, MD, ${ }^{a}$ Chunli Jiang, MD, ${ }^{a}$ Shintaro Nemoto, MD, PhD, ${ }^{a}$ Yoshiaki Saji, MD, ${ }^{\text {a }}$ Tadashi Ikeda, MD, PhD, Shinichi Urayama, MS, ${ }^{b}$ Takashi Azuma, BS, ${ }^{c}$ Akishige Hokugo, DDS, PhD, ${ }^{c}$ Sadami Tsutsumi, PhD, ${ }^{c}$ Yasuhiko Tabata, PhD, DMedSc, DPharm, ${ }^{\mathrm{c}}$ and Masashi Komeda, MD, PhDa

Supplemental material is available online.
From the Department of Cardiovascular Surgery, ${ }^{\mathrm{a}}$ Human Brain Research Center, ${ }^{\mathrm{b}}$ Kyoto University, Graduate School of Medicine, Kyoto, Japan, and the Institute for Frontier Medical Sciences, ${ }^{\mathrm{c}}$ Kyoto University, Kyoto, Japan.

This study was supported in part by a grantin-aid for scientific research from the Ministry of Education, Culture, Sports, Science and Technology (\#17591468), a grant-inaid for cardiovascular disease research from the Japan Heart Foundation/Pfizer Japan Pharmaceutical, and a grant-in-aid for cardiac disease research from the Vehicle Racing Commemorative Foundation.

Read at the Eighty-fifth Annual Meeting of The American Association for Thoracic Surgery, San Francisco, Calif, April 10-13, 2005.

Received for publication Dec 22, 2005; revisions received June 5, 2006; accepted for publication June 22, 2006.

Address for reprints: Masashi Komeda, MD, PhD, Professor and Chairman, Department of Cardiovascular Surgery, Graduate School of Medicine, Kyoto University, 54 Shogoin Kawahara-machi, Sakyo-ku, Kyoto 606-8507, Japan (E-mail: komelab@kuhp. kyoto-u.ac.jp).

J Thorac Cardiovasc Surg 2006;132:891-9

$0022-5223 / \$ 32.00$

Copyright (c) 2006 by The American Association for Thoracic Surgery

doi:10.1016/j.jtcvs.2006.06.023
Objective: The purpose of this study was to evaluate the therapeutic efficacy of the combined growth factor therapy with an omental flap in a rabbit model of chronic myocardial ischemia.

Methods: Chronic ischemia was created in rabbits by placing a constrictor on the left circumflex artery. Four weeks later the animals were divided into 3 groups: group $\mathrm{FG}$, in which a gelatin hydrogel sheet incorporating $100 \mu \mathrm{g}$ of basic fibroblast growth factor was placed over the left circumflex region followed by covering with the omental flap including the intact gastroepiploic artery; group F, in which only the basic fibroblast growth factor sheet was placed; and group $\mathrm{N}$, in which no treatment was done.

Results: Cine magnetic resonance imaging analysis showed a greater percentage wall thickening in the left circumflex region in group FG than in other groups (group FG, $49.2 \% \pm 4.5 \%$; group $\mathrm{F}, 41.2 \% \pm 3.8 \%$; group $\mathrm{N}, 32.1 \% \pm 2.5 \%, P=.035$, group FG vs group F). A colored microsphere assay showed higher perfusion in the left circumflex region in group FG than in group F. Perfusion in the left circumflex region was decreased after clamping the gastroepiploic artery pedicle in group FG (before clamping, $2.83 \pm 0.72 \mathrm{~mL} \cdot \min ^{-1} \cdot \mathrm{g}^{-1}$; after clamping, $1.93 \pm 0.59 \mathrm{~mL}$ $\left.\cdot \min ^{-1} \cdot \mathrm{g}^{-1} ; P<.01\right)$. In vivo angiography via gastroepiploic artery showed direct "to-and-fro" visible collaterals between the gastroepiploic and occluded left circumflex coronary arteries in group FG.

Conclusion: The combined growth factor therapy with an omental flap induced arteriogenesis and provided additional perfusion via the gastroepiploic artery to ameliorate regional dysfunction in the chronically ischemic myocardium.

$\mathrm{D}$ espite advances in the treatment for ischemic heart disease, there exist patients who are not eligible for current revascularization procedures because of chronic, diffuse, and poorly graftable coronary lesions. ${ }^{1}$

As progress has been made in the basic studies on growth factors in the normal angiogenic process, the concept of therapeutic angiogenesis was developed as an alternative treatment for these patients over the past 2 decades. $^{2}$ Preclinical animal studies with various growth factor delivery strategies have shown promising data. ${ }^{3,4}$ However, recent randomized double-blind clinical trials showed disappointing results with respect to therapeutic efficacy., 5

Historically, before the advancement of cardiopulmonary bypass, the concept of employing an omental flap to provide revascularization for the ischemic myocardium was attempted in patients with ischemic heart disease. However, the thera- 

Abbreviations and Acronyms
$\mathrm{bFGF}=$ basic fibroblast growth factor
FS $\quad=$ fractional shortening
GEA = gastroepiploic artery
LCx = left circumflex coronary artery
$\mathrm{LV}=$ left ventricular
LVEDD $=$ left ventricular end-diastolic dimension
LVEF = left ventricular ejection fraction
LVESD = left ventricular end-systolic dimension
MRI = magnetic resonance imaging

peutic efficacy of omentopexy was not so efficient for rapid recovery. There exist the clinical limitations with the therapeutic efficacy of ometopexy as well as with that of growth factor therapy. ${ }^{7}$

To offer a more effective therapeutic option for these patients, we developed a combined method involving an omental flap and growth factor as a new alternative surgical method. We called this strategy "biologic coronary artery bypass grafting." Our previous study demonstrated that the method augmented the therapeutic efficacy of growth factor therapy using sustained-release basic fibroblast growth factor (bFGF) by applying a pedicled omental flap in a rabbit model of acute myocardial infarction, whereas omentopexy alone was not so effective as growth factor therapy. ${ }^{8} \mathrm{Re}-$ garding the clinical application, the purpose of the present study was to evaluate its therapeutic efficacy and to verify its superior therapeutic effect over growth factor therapy in the chronically ischemic myocardium.

\section{Materials and Methods}

\section{Experimental Animals and Study Protocol}

Forty adult male white Japanese rabbits (weighing 3.5-4.0 kg) (Shizuoka Laboratory Animal Center, Shizuoka, Japan) were used in this study. All the animal experiments in this study were performed according to the institutional guidelines on animal experimentation of Kyoto University, which conform to the "Guidance for the Care and Use of Laboratory Animals" law in Japan.

Each animal received two consecutive operations during the study. In the first operation, we created chronic myocardial ischemia by placing an ameroid constrictor. Four weeks after the first operation, animals were assigned into 3 groups as follows: group $\mathrm{N}(\mathrm{n}=8)$ received no additional treatment after creation of the myocardial ischemia; in group $\mathrm{F}(\mathrm{n}=8)$ a gelatin hydrogel sheet incorporating $100 \mu \mathrm{g}$ of bFGF was placed over the epicardium of the ischemic area; and in group FG $(n=8)$ a gelatin hydrogel sheet incorporating $100 \mu \mathrm{g}$ of bFGF was placed over the ischemic area, followed by covering with an omental flap. Four weeks after the second operation, animals were put to death with an overdose of pentobarbital to harvest the cardiac tissue for further assessments.

Anesthetic protocol for the surgical procedures and functional measurements. All surgical procedures in this study were performed with the animals under general anesthesia as described below. The rabbits were sedated with an intravenous injection of sodium pentobarbital $(30 \mathrm{mg} / \mathrm{kg})$ and then intubated with an endtracheal tube (4.0-mm inner diameter, neonatal end-tracheal tube; Mallinckrodt Medical, St Louis, Mo) for mechanical ventilation (tidal volume of 10-15 mL and a minute ventilation rate of 40-60 breaths/min) (7025 Rodent Ventilator; Ugo Basile, Rome, Italy). General anesthesia was maintained with $1.0 \%$ to $2.0 \%$ of isoflurane mixed with room air. Rectal temperature was maintained at $38^{\circ} \mathrm{C}-39^{\circ} \mathrm{C}$ with a heat pad during the surgical procedures.

\section{Creation of Chronic Myocardial Ischemia With an Ameroid Constrictor: The First Operation}

We used a rabbit model of chronic myocardial ischemia according to the method previously described by Operschall and coworkers. ${ }^{9}$ In brief, a left thoracotomy was performed at the fourth intercostal space in a sterile manner. After the pericardium was opened, the main branch of the left circumflex (LCx) coronary artery was identified. We applied a commercially available hygroscopic ameroid constrictor specially designed for constricting rabbit coronary arteries (5-mm diameter, 1.5-mm height: Research Instruments SW, San Diego, Calif). We fixed it on the epicardial surface of the heart, surrounding the targeted coronary artery, with a 6-0 polypropylene suture placed around its circumference. The knot was softly tied to place the ameroid constrictor on the epicardial surface of the targeted artery preventing complete occlusion of the flow, as demonstrated by both electrocardiographic changes and visual blanching of the myocardium. Careful inspection was maintained for 10 minutes after placement of the constrictor. The thoracotomy was closed in layers, and residual air in the thoracic cavity was evacuated.

Echocardiographic measurements of time-course cardiac function. Left ventricular (LV) function was assessed by transthoracic echocardiography at each procedure (every 4 weeks) under general anesthesia as described above. A commercially available echocardiograph with a $7.5-\mathrm{MHz}$ pediatric transducer (Vivid 7; GE Medical, Tokyo, Japan) was used for all studies to obtain serial images through a left parasternal approach. LV enddiastolic and end-systolic dimensions (LVEDD and LVESD, respectively) were measured with M-mode tracings from the shortaxis view at the papillary muscle level. Fractional shorting (FS) was calculated from these data as follows: FS $(\%)=($ LVEDD LVESD $) /($ LVEDD $) \times 100$. The data were averaged over 3 consecutive cardiac cycles. Another observer who was blinded to the treatment groups performed all measurements.

Preparation of the gelatin hydrogel sheet incorporating bFGF. Gelatin hydrogel sheets (Nitta Gelatin Co, Osaka, Japan) were prepared as described previously. ${ }^{10}$ In brief, the sheets were freeze-dried and trimmed in $5 \times 5-\mathrm{mm}$ squares and $0.7-\mathrm{mm}$ thick, then impregnated with an aqueous solution containing $100 \mu \mathrm{g}$ of human recombinant bFGF (Kaken Pharmaceutical Co, Tokyo, Japan). All the processes were conducted under sterile conditions.

\section{Treatment: The Second Operation}

Four weeks after the first operation, each animal in groups FG and F received 2 different types of treatment as the second operation. We excluded rabbits if they showed an FS more than $30 \%$ or less than $15 \%$ in the echocardiograhic assessment before the second operation. 
In group $\mathrm{F}$, a median sternotomy was performed to place the bFGF-incorporated hydrogel sheet (bFGF sheet) on the epicardium of the ischemic area (LCx region) by stitching around the edge of the sheet. In group FG, a small upper midline laparotomy and median sternotomy were performed to take the omentum out from the peritoneal space into the mediastinal space, preserving the arch structure of the left gastroepiploic artery (GEA). We created the hole at the diaphragm and passed the omental flap through the hole into the pericardial cavity. The bFGF sheet was placed on the epicardium of the ischemic area, followed by covering the sheet with the harvested omental flap.

\section{Magnetic Resonance Imaging Analysis of Cardiac Performance}

Four weeks after the second operation, we performed electrocardiographically gated cine magnetic resonance imaging (MRI) scans (Siemens Sonata 1.5 Tesla; Siemens Medical System, Erlangen, Germany) while the animals were anesthetized with an intravenous injection of sodium pentobarbital $(30 \mathrm{mg} / \mathrm{kg})$. A circular polarized extremity coil was wrapped around the chest for signal acquisition. Six sequential images of LV short-axis views every $2.5 \mathrm{~mm}$ were obtained to cover the entire LV volume from the base to the apex over 10 heartbeats. Custom designed software (Image J 1.3 version; Scion Corporation, Frederick, Md) was used to define myocardial borders and measure the wall thickness in the LCx region by the modified centerline method. ${ }^{11}$ The circumferential lengths at end-diastole and end-systole at the papillary muscle level were measured. LV volumes at end-systolic and end-diastolic phase (LVESV and LVEDV, respectively) were computed by the area-length method and used to calculate the LV ejection fraction $(\mathrm{LVEF})$ as follows: $\operatorname{LVEF}(\%)=(\mathrm{LVEDV}-$ LVESV)/LVEDV $\times 100 .{ }^{12}$

\section{Measurement of Regional Myocardial Blood Flow}

Four weeks after the second operation, we evaluated regional myocardial blood flow in the ischemic area (LCx region) with a colored microsphere technique (DYE-TRCK; Triton Technology, Toronto, Ontario, Canada). A repeated left thoracotomy was performed to expose the left atrial appendage. After systemic heparinization (1000 IU heparin), 1.2 million red colored microspheres (15 $\mu \mathrm{m}$ in diameter) were injected into the left atrium for more than 30 seconds while reference blood samples were drawn from the descending aorta at a rate of $1.0 \mathrm{~mL} / \mathrm{min}$ for 90 seconds. In group FG, 1.2 million yellow colored microspheres were consecutively injected to evaluate the GEA flow into the ischemic area in the same manner after clamping the GEA pedicle at the level of the diaphragm through a small laparotomy. After euthanasia with an overdose of pentobarbital, the LV was isolated and 3 short-axis transverse slices were cut out, while the transverse slices at the midventricular level were cut into 4 radial segments. According to the manufacturer's protocol, we extracted microspheres by potassium hydroxide digestion from the lateral midventricular segments and blood samples. The dyes were extracted from the spheres with $100 \mu \mathrm{L}$ of dimethylformamide, and their concentrations were determined by spectrophotometry (UV-mini 1240; Shimazu. Co, Kyoto, Japan). The myocardial blood flow (Qs) was calculated as follows: Qs $\left(\mathrm{mL} \cdot \min ^{-1} \cdot \mathrm{g}^{-1}\right)=(\mathrm{As} / \mathrm{Ar}) \mathrm{Qr}(\mathrm{mL} / \mathrm{min}) / \mathrm{Wt}(\mathrm{g})$, where $\mathrm{Qr}$ represents with the withdrawal rate of the reference blood, As and Ar represent the absorbance in the sample tissue and reference blood, and Wt represents the tissue weight.

\section{Histologic Analysis}

Four weeks after the second operation, another series of 6 rabbits in each group was used for histologic analysis. After euthanasia, the heart was perfused at $100 \mathrm{~mm} \mathrm{Hg}$ for 10 minutes with $10 \%$ formalin and then immersion-fixed in $4 \%$ formalin. We isolated the LV and cut out the short-axis transverse slice corresponding to the ischemic region situated $2 \mathrm{~mm}$ below the implanted ameroid constrictor. This transverse slice of LV at the papillary muscle level was embedded in paraffin and sectioned at $4-\mu \mathrm{m}$ thickness. The primary mouse monoclonal antibody against $\alpha$-smooth muscle actin (clone 1A4; Sigma Chemical Co, St Louis, Mo) was incubated with the tissue section, followed by incubation with a biotin-rabbit anti-mouse immunoglobulin G. Tetramethylrhodamine isothiocyanate-conjugated secondary antibody was used to detect expression of $\alpha$-smooth muscle actin. The tissue sections were counterstained with hematoxylin and eosin. The numbers of arterioles were counted under a microscopic field $(\times 100)$ to determine the arteriolar density. Five high-power fields were randomly selected for the vessel counts at the center of the lateral myocardial territories in each section. An arteriole was defined as a vessel in diameter more than $50 \mu \mathrm{m}$. Quantification was performed in a blinded manner with a minimum of 3 sections for each animal.

\section{Microvascular Corrosion Cast}

We made a microvascular corrosion cast to evaluate 3-dimensional collateral development in group FG, as previously described. ${ }^{13}$ Four weeks after the second operation, the resin (Mercox CL; Dainippon Ink Chemical, Tokyo, Japan) was injected antegradely into the celiac arterial trunk and retrogradely into the descending thoracic aorta after systemic heparinization (1000 IU heparin). The whole body was immersed in hot water for a few hours to solidify the resin in the blood vessels. The heart covered with the omental flap was carefully harvested and then placed into $10 \%$ sodium chlorate solution to corrode the residual adjacent tissue except for the arteries. This specimen was fixed with liquid nitrogen. After being coated with platinum-palladium, an image of collateral vessels was obtained by scanning electron microscopy (S4000; Hitachi Co, Tokyo, Japan)

\section{In Vivo Angiography}

A different series of 5 rabbits in group FG were anesthetized as described above. The right common carotid artery was cannulated with a 4F sheath introducer system (Goodtech; Goodman Co, Nagoya, Japan) after systemic heparinization (1000 IU heparin). A 3.2F catheter (Selecon PA catheter; Clinical Supply Co, Gifu, Japan) was selectively inserted into the celiac arterial trunk under fluoroscopy (OEC9800; GE Medical, Tokyo, Japan). The serial images of the collateral arteries were recorded at the rate of 30 frames per second with manual injection of 10 to $30 \mathrm{~mL}$ of diluted nonionic contrast medium (Iopamiron 300; Schering Co, Munich, Germany). Collateralization and myocardial blush were assessed qualitatively.

\section{Statistical Analysis}

All the data are shown as mean \pm standard deviation. Statistical analyses were performed with the Stat-View software (SAS Insti- 
tute Inc, Cary, NC). Comparisons of echocardiographic data among the groups were performed by 2 -way repeated measures analysis of variance. Comparisons of other data among the groups were performed by 1 -way analysis of variance. If significance was found for a group, a time effect, or a group-by-time interaction, differences between groups were specified with the Tukey-Kramer test for post hoc comparisons.

\section{Results}

Feasibility

There was no periprocedural mortality. Two rabbits in group $\mathrm{N}$ died at 5 weeks after the first operation with evidence of lateral myocardial infarction at autopsy.

Time-course Changes of Global LV Function Assessed by Serial Transthoracic Echocardiography

Four weeks after the constrictor implantation, FS was significantly reduced in all groups. Four weeks after each treatment, group FG showed a greater FS increase than group $\mathrm{F}(P=.038$ vs group $\mathrm{F}$ ) (Figure $1, A)$. Four weeks after the constrictor implantation, LVEDD was increased in all groups. Four weeks after each treatment, group FG and group $\mathrm{F}$ showed a greater recovery in LVEDD than group $\mathrm{N}$, but there was no significant difference between group $\mathrm{FG}$ and $\mathrm{F}$ $(P=.143$ vs group $\mathrm{F}, P=.008$ vs group $\mathrm{N})($ Figure $1, B)$.

\section{Assessment of Regional and Global LV Function by Cine MRI}

Four weeks after each treatment, percentage wall thickening in the LCx region was significantly higher in group FG than in groups $\mathrm{F}$ and $\mathrm{N}(P=.035$ vs group $\mathrm{F})$ (Figure $2, B)$. Although there was no significant difference in the circumferential length at end-diastole between groups $\mathrm{F}$ and FG, circumferential length at end-systole was significantly well maintained in group FG compared with group F. LVEF was significantly higher in group FG than in group F (Table E1).

Assessment of Regional Myocardial Blood Flow in the Ischemic Region Using the Colored

Microsphere Technique

Four weeks after each treatment, regional myocardial blood flow in the LCx region was significantly higher in group FG than in group $\mathrm{F}(P=.035$ vs group $\mathrm{F}$ ) (Figure $3, A)$. In group $\mathrm{FG}$, the regional myocardial blood flow in the $\mathrm{LCx}$ region was significantly decreased after the GEA pedicle was clamped $(P=.008)$ (Figure 3, $B)$.

Immunnohistochemical Analysis of Neoarterial Formation in the Ischemic Region

A significantly greater number of arterioles in the $\mathrm{LCx}$ region were identified in group $\mathrm{FG}$ than in group $\mathrm{F}(P=$ .048 vs group F) (Figure 4, B).
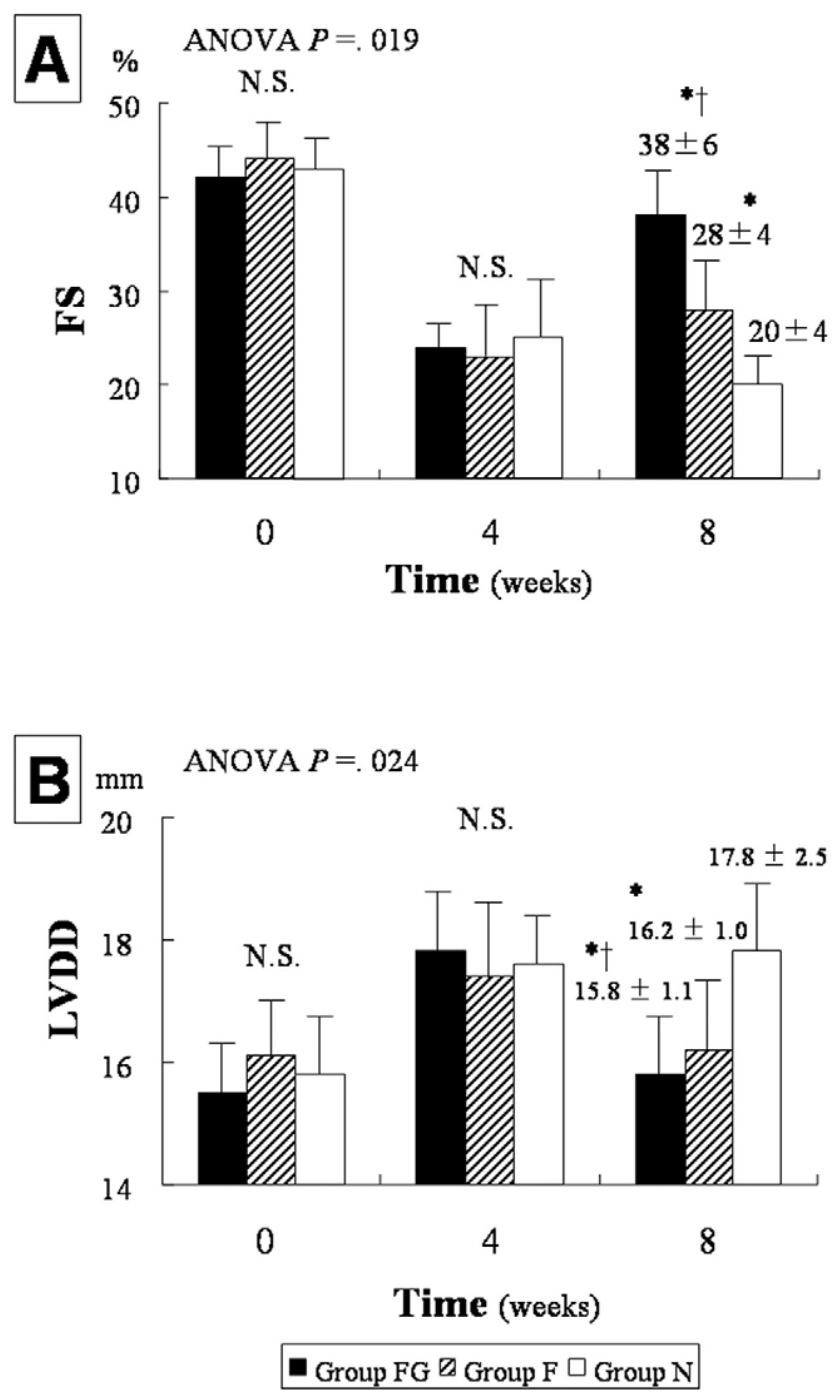

Figure 1. Time course changes of global LV function assessed by echocardiography. FS (A) and LVEDD (B) assessed before placement of a constrictor (baseline: 0 week) and at treatment (4 weeks) and 4 weeks after treatment (8 weeks). Asterisk indicates $\boldsymbol{P}<.01$ versus group $\mathrm{N}$; dagger indicates $\boldsymbol{P}<.05$ versus group F. N.S, Not significant.

Three-Dimensional Assessment of Collateral Vessels by Scanning Electron Microscopy in the Microvascular Corrosion Cast Specimen

The corrosion cast specimen from group FG showed marked collateral formation between the GEA and native coronary arterial branches (Figure 5,A). The macroscopically visible collateral vessels were easily identified (Figure 5, B). Furthermore, scanning electron microscopic analysis disclosed that the diameter of these collateral arteries was more than 150 $\mu \mathrm{m}$ (Figure 5, C). 


\section{End-Systole}

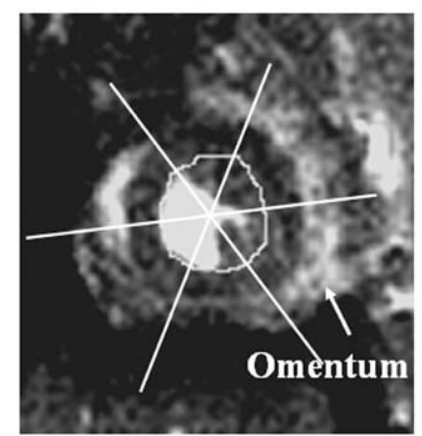

B

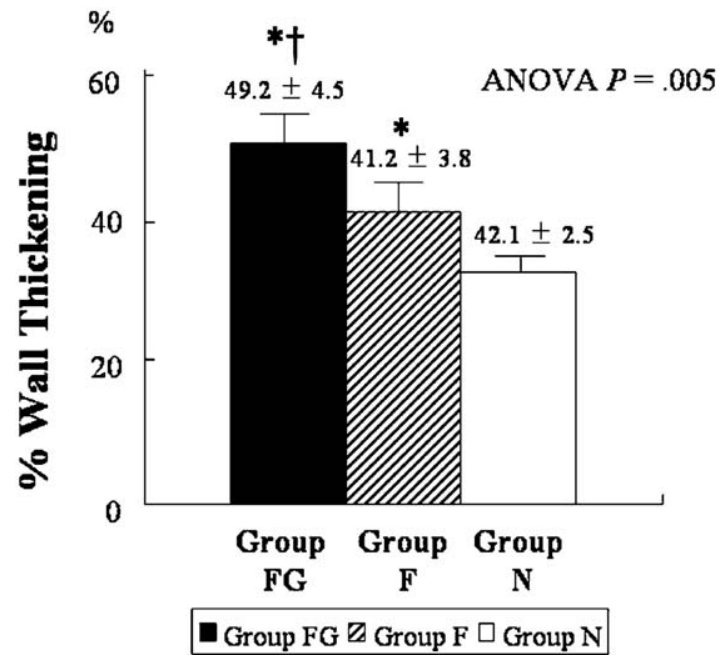

Figure 2. Regional LV function in the ischemic region assessed by cine MRI. A, Representative cine MRI images of a short-axis view at the papillary muscle level in group FG at 4 weeks after treatment. Regional myocardial thickening assessed by the modified centerline method; changes in length of cords between end-diastole and end-systole determine wall thickening. $R V$, Right ventricular cavity; $L V$, left ventricular cavity; $\boldsymbol{C x}_{x}$, circumflex artery. B, Percent wall thickening in the ischemic region assessed by cine MRI analysis at 4 weeks after treatment in each group. Asterisk indicates $P<.01$ versus group N; dagger indicates $\boldsymbol{P}<.05$ versus group $\mathrm{F}$.

Angiographic Assessment of the Collateral Communication Between the GEA and the Native Occluded LCx Artery

In group FG, communication between the GEA and native coronary arterial branches was identified in all animals. We found direct "to-and-fro" communications between the GEA and the proximally occluded LCx artery in 3 animals (see Video). In another 2 animals, direct opacification of the proximally occluded LCx artery was not demonstrated, but delayed

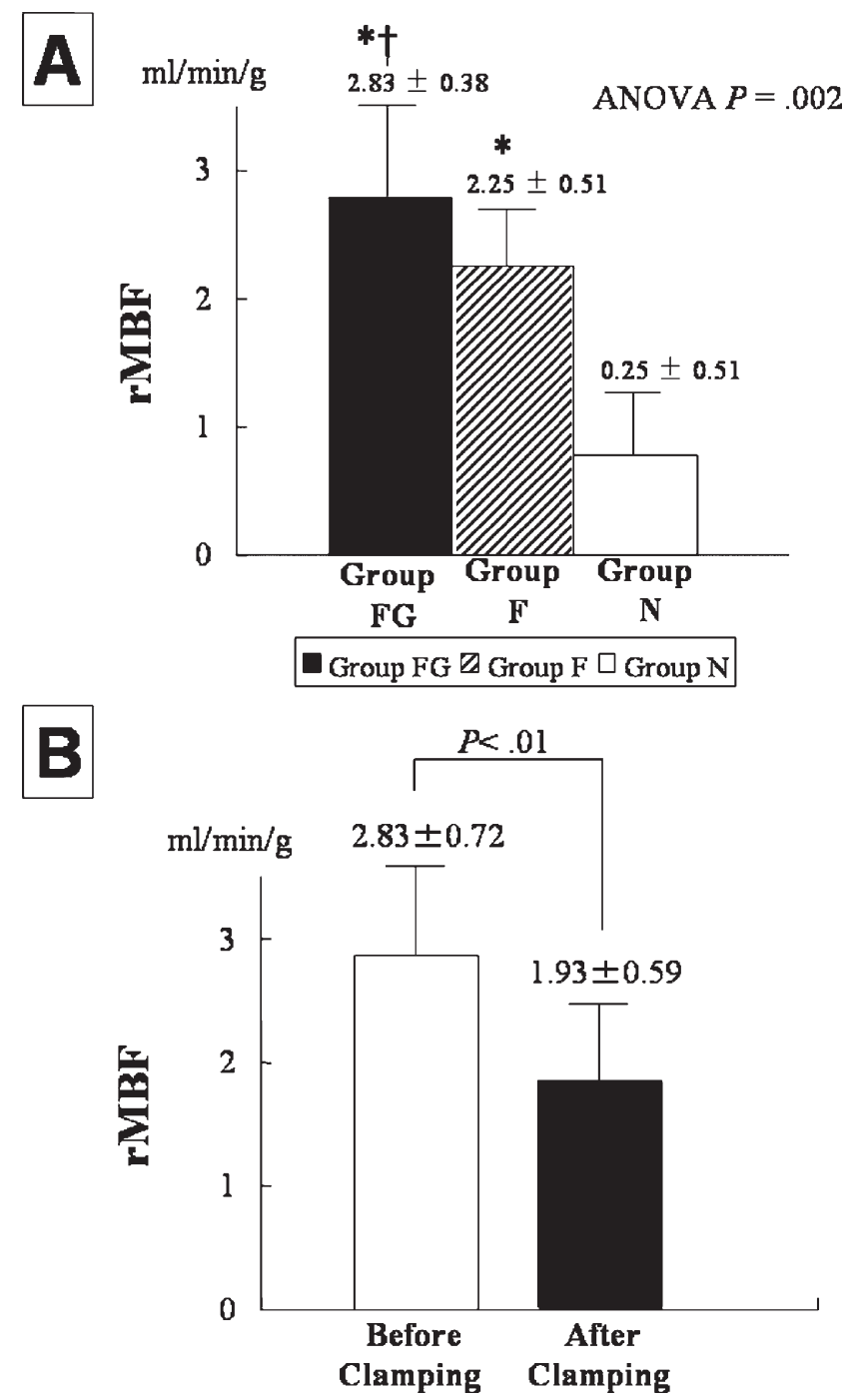

Figure 3. Regional myocardial blood flow (rMBF) in the LCx region assessed by the colored microsphere technique. $A$, rMBF in $\mathrm{LCx}$ region at 4 weeks after each treatment assessed by colored microsphere technique. Asterisk indicates $\boldsymbol{P}<.01$ versus group $N$; dagger indicates $\boldsymbol{P}<.05$ versus group $\mathrm{F}$. $\mathrm{B}$, The change of $\mathrm{rMBF}$ in the $\mathrm{LCx}$ region in group FG before and after clamping the GEA pedicle. Asterisk indicates $P<.01$. ANOVA, Analysis of variance.

opacification through the marked collaterals from the GEA was easily identified.

\section{Discussion}

In the present study, we demonstrated the therapeutic effects of the combined method involving an omental flap including the GEA and single growth factor therapy using a bFGF sustained-releasing biodegradable sheet. The method developed collateral vessels directly from the GEA and provided 
additional perfusion via the GEA to improve regional LV dysfunction resulting from chronic ischemia. Furthermore, the combined method showed its superior therapeutic effect over single growth factor therapy in the chronically ischemic myocardium.

The microsphere assay in the present study showed that the combined method provided more perfusion in the ischemic region than single growth factor therapy. Moreover, in the group treated by the combined method, we observed $32 \%$ reduction of the perfusion in the ischemic region after clamping the GEA. These results clearly demonstrated that the combined method provided additional perfusion to the ischemic region via the GEA. In the cine MRI analysis, the combined method showed a marked improvement in regional contractility in the ischemic region as expressed in percentage wall thickening, which is referred to as an index of mechanical function across all layers of the myocardium. Generally, providing adequate perfusion to all myocardial layers in the ischemic region is needed to improve regional dysfunction caused by ischemia. ${ }^{14}$ These findings demonstrated that the combined method can provide additional blood flow through the GEA to perfuse all myocardial layers of the region of chronic ischemia.

Perfusion reflects the result of successful collateral formation. The development of effective collateral vessels involves arteriogenesis, which is referred to as a process based on growth and remodeling into functional vessels. ${ }^{15}$ Arteriogenesis is considered to be more important than angiogenesis due to higher perfusion capacity. ${ }^{16}$ As the morphologic parameter for arteriogenesis, the immunohistologic analysis in the present study showed that the combined method developed more arterioles in the ischemic region compared with single growth factor therapy. In addition, the combined method developed sizable collateral vessels directly from the GEA, as shown in the vascular corrosion cast study. These results suggest that the combined method induced arteriogenesis to develop the collateral vessels directly from the GEA in the chronically ischemic myocardium.

As described in our previous study, our strategy for developing effective collaterals for advanced coronary lesions is to apply an omental flap involving the GEA as nondiseased donor artery with high perfusion capacity. ${ }^{8}$ The process of arteriogenesis is mediated via shear stress. ${ }^{15}$ The intracoronary collaterals induced by single growth factor therapy provide the inadequate limited flow through the obstructed native donor coronary arteries to induce arteriogenesis for developing effective collateral vessels. ${ }^{17}$ In contrast, an omental flap can provide the adequate additional flow via the GEA as an unobstructed donor artery. From these considerations, the GEA involved in an omental flap can play an important role as an independent, unobstructed extracardiac blood source to provide additional collateral

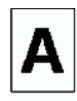

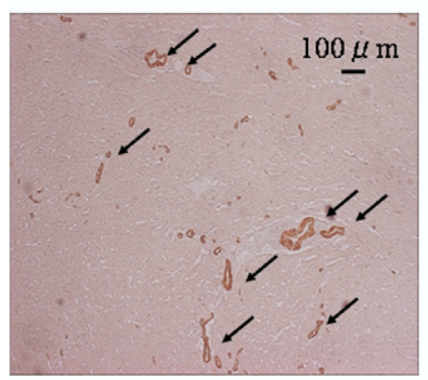

Group FG

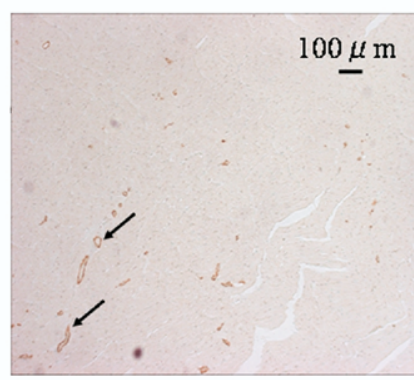

Group F
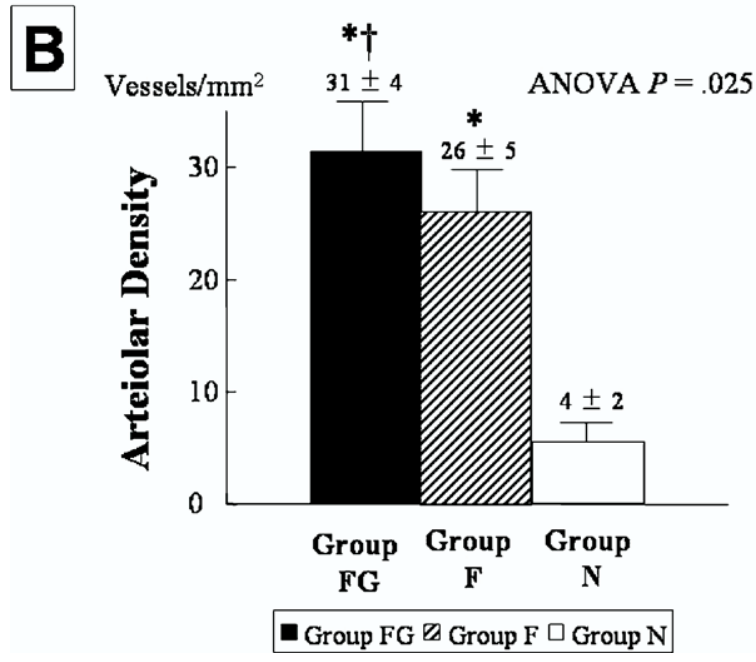

Figure 4. Histologic assessment of arteriogenesis in the ischemic myocardium after treatment. A, Representative images of immunohistochemical staining in group $F$ and group FG. Arterioles in the LCx region were stained with $\alpha$-smooth muscle actin antibody. Arrows indicate arterioles with a diameter more than $\mathbf{5 0}$ $\boldsymbol{\mu m}$. Scale bar indicates $\mathbf{1 0 0} \boldsymbol{\mu m}$ in length (original magnification $\times 100$ ). B, Quantification of the number of arterioles with a diameter more than $50 \mu \mathrm{m}$ in each group. Asterisk indicates $P<.01$ versus group N; dagger indicates $P<.05$ versus group $\mathrm{F}$. ANOVA, Analysis of variance.

flow (shear stress) for enhancing arteriogenic activity in the chronically ischemic region.

Conversely, from the results of our studies, it is reasonable to conjecture that the combined method may enhance the angiogenic efficacy of omentopexy by interposing the bFGF sheet. As demonstrated by our previous study, the therapeutic efficacy of omentopexy alone was inferior to that of single growth factor therapy with bFGF sheet. ${ }^{8}$ Five decades ago, omentopexy was used to create collaterals from the GEA. However, in some cases, it took several years to develop effective ones. ${ }^{18}$ These facts suggest that 

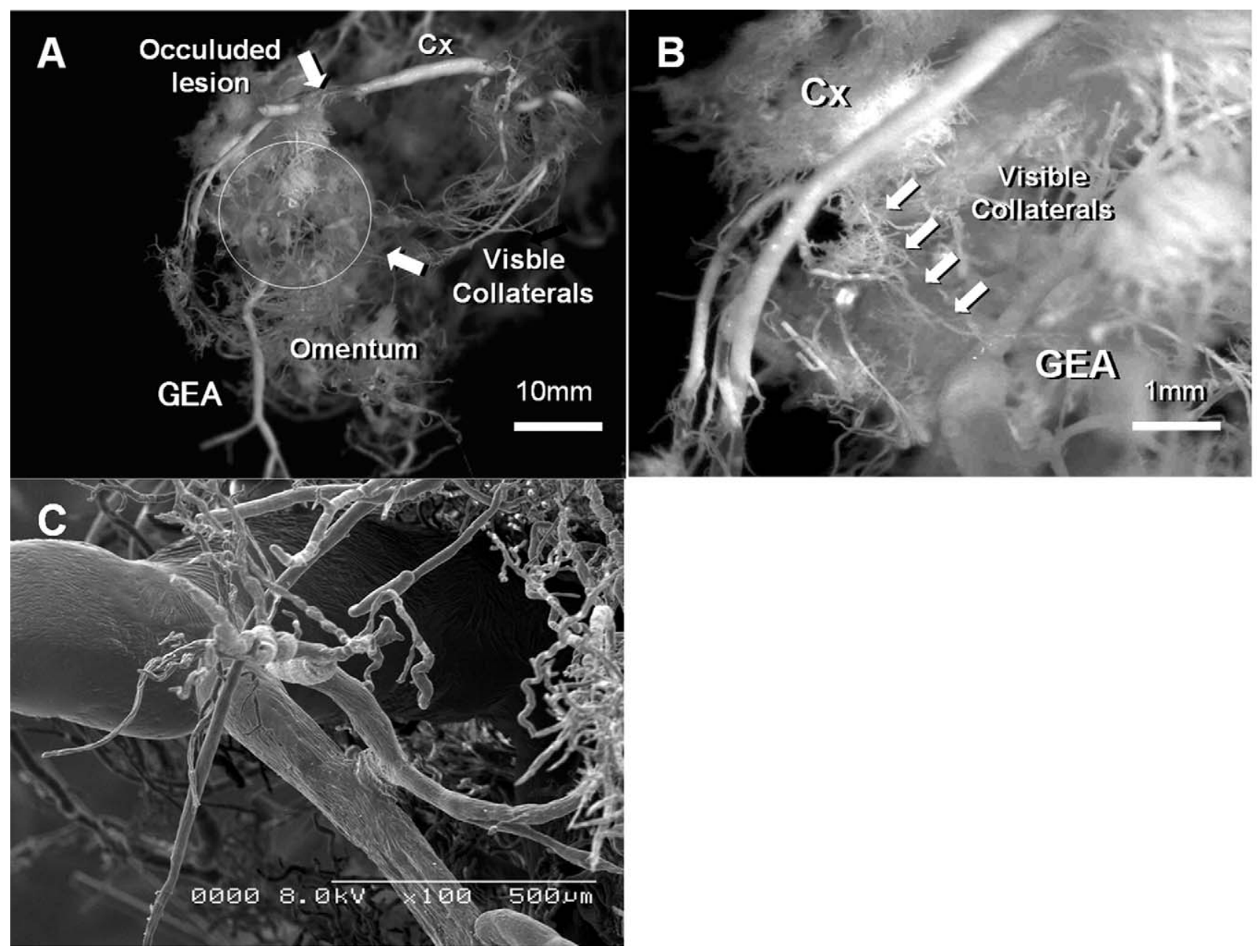

Figure 5. Microvascular corosion cast image of collateral formation in group FG. A, The whole specimen. Representative image of a microvascular corosion cast specimen in group FG. Scale bar indicates $10 \mathrm{~mm}$ in length (original magnification $\times 6$ ). $C x$, Left circumflex artery; $G E A$, gastroepiploic artery. $B$, Representative image of collateral formation between distally occluded LCx and GEA. Scale bar indicates $1 \mathrm{~mm}$ in length. (Original magnification $\times 60$ ). C, Scanning electron micrographs of collaterals between distally occluded LCx and GEA in the microvascular corrosion cast specimens.

the clinical disadvantage of omentopexy is its therapeutic inefficiency. The interposed bFGF sheet possibly enhances the angiogenic effects of the superimposed omental flap in the combined method. It can accelerate the formation of collaterals between native coronary arteries and omental tissue to achieve the vessel connections directly from the GEA.

Omental flap itself has been an attractive tissue for cardiothoracic surgeons to stimulate revascularization of ischemic tissue. ${ }^{19}$ Recent basic studies have elucidated the mechanism of the angiogenic action induced by omental tissue. The adipocytes in the omental tissue release a number of angiogenic growth factors, such as vascular endothelial growth factor and bFGF. This fact suggests that an omental flap can act as a physiologic exogenous source of multiangiogenic factors that are synergistically involved in the process of arteriogenesis. ${ }^{20,21}$ Moreover, the interposed bFGF sheet possibly can augment the expression of other growth factors in the omental tissue. ${ }^{22}$ However, further investigation is needed to clarify the participation of the additive growth factors released from the omental tissue in the process of collateral formation from the GEA in the combined method.

Other experimental studies were reported to enhance the effects of omentopexy. Ruel and associates ${ }^{23}$ demonstrated the excellent angiogenic effect of a gastric submucosal patch as an endogenous source of growth factors in a swine model of chronic myocardial ischemia. Kanamori and col- 
leagues ${ }^{24}$ showed that omentopexy enhanced the angiogenic effect of cell therapy in a swine model of acute myocardial infarction. Compared with these studies, the combined method in the present study is safer and less invasive because it does not need a gastrectomy or bone marrow aspiration.

Several limitations of this study must be addressed. First, we did not quantitatively evaluate the functional capacity of developed collaterals in this study. However, the identification of angiographically visible "to-and-fro" collaterals can be considered as the qualitative evidence of functional collateral formation. Second, there existed a variation in the myocardial ischemia in the animal model of chronic ischemia with an Ameroid constrictor, although we designed the study to minimize this variation. As a further study, the efficacy of the method must be evaluated in hyperlipidemic or diabetic animal models because endothelial dysfunction resulting from these pathologic factors is one of the most important obstacles to achieving clinical therapeutic angiogenesis. ${ }^{25,26}$

In conclusion, we demonstrated that the combined method developed sizable collateral vessels directly from the GEA to ameliorate regional myocardial dysfunction in the chronically ischemic myocardium. We verified that the effect of the present method is superior to that of single angiogenic protein therapy. The present method may improve the clinical limitations in therapeutic angiogenesis and expand the surgical indication for patients with severe coronary artery disease.

We thank Toshiya Sato, MD, $\mathrm{PhD}$, for the instructions of statistical analysis, Shinji Tomita, $\mathrm{MD}, \mathrm{PhD}$, for the reviewing our manuscript, and Mrs Fumiyo Kataoka for her assistance in histologic assessment.

\section{References}

1. Mukherjee D, Comella K, Bhatt DL, Roe MT, Patel V, Ellis SG, et al Clinical outcome of a cohort of patients eligible for therapeutic angiogenesis or transmyocardial revascularization. Am Heart J. 2001;42: $72-4$.

2. Folkman J. Tumor angiogenesis: therapeutic implications. $N$ Engl J Med. 1971;285:1182-6.

3. Mack CA, Patel SR, Schwarz EA, Zanzonico P, Hahn RT, Ilercil A, et al. Biologic bypass with the use of adenovirus-mediated gene transfer of the complementary deoxyribonucleic acid for vascular endothelial growth factor 121 improves myocardial perfusion and function in the ischemic porcine heart. $J$ Thorac Cardiovasc Surg. 1998;115:168-76.

4. Harada K, Grossman W, Friedman M, Edelman ER, Prasad PV, Keighley C, et al. Basic fibroblast growth factor improves myocardial function in chronically ischemic porcine hearts. J Clin Invest. 1994; 94:623-30.

5. Simons MS, Annex BH, Laham RJ, Kleiman N, Henry T, Dauerman $\mathrm{H}$, et al. Pharmacological treatment of coronary artery disease with recombinant fibroblast growth factor-2: double blind, randomized, controlled clinical trial. Circulation. 2002;105:788-93.

6. Ruel M, Song J, Sellke FW. Protein-, gene-, and cell-based therapeutic angiogenesis for the treatment of myocardial ischemia. Mol Cell Biochem. 2004;264:119-31.

7. O'Shaugnessy L. Surgical treatment of cardiac ischemia. Lancet. 1937;232:185-94.
8. Ueyama K, Bing G, Tabata Y, Ozeki M, Doi K, Nishimura K, et al Development of biologic coronary artery bypass grafting in a rabbit model: revival of a classic concept with modern biotechnology. $J$ Thorac Cardiovasc Surg. 2004;127:1608-15.

9. Operschall C, Loretta F, Jean-Paul C, Se'bastien R. A new model of chronic cardiac ischemia in rabbits. $J$ Appl Physiol. 2000;88:1438-45.

10. Iwakura A, Tabata Y, Tamura N, Doi K, Nishimura K, Nakamura T, et al. Gelatin sheet incorporating basic fibroblast growth factor enhances healing of devascularized sternum in diabetic rats. Circulation. 2001;104:325-9.

11. Sheehan FH, Bolson EL, Dodge HT, Mathey DG, Schofer J, Woo HW Advantages and applications of the centerline method for characterizing regional ventricular function. Circulation. 1986:74:293-305.

12. Lawson MA, Blackwell GG, Davis ND, Roney M, Dell'Itallia LJ, Pohost GM. Accuracy of biplane long axis left ventricular volume determined by cine magnetic resonance imaging in patients with regional and global dysfunction. Am J Cardiol. 1996;77:1098-104.

13. Hokugo A, Kubo Y, Takahashi Y, Fukuda A, Horiuchi K, Mushimoto K, et al. Prefabrication of vascularized bone graft using guided bone regeneration. Tissue Eng. 2004;10:978-86.

14. Schulz R, Heusch G. The relationship between regional blood flow and contractile function in normal, ischemic, and reperfused myocardium. Basic Res Cardiol. 1998;93:455-62.

15. Heil M, Schaper W. Influence of mechanical, cellular, and molecular factors on collateral artery growth (arteriogenesis). Circ Res. 2004;95: 449-58.

16. Royen N, Piek JJ, Buschmann I, Hoefer I, Voskuil M, Schaper W. Stimulation of arteriogenesis; a new concept for the treatment of arterial occlusive disease. Cardiovasc Res. 2001;49:543-53.

17. Unger EF, Sheffield CD, Epstein SE. Creation of anastomoses between an extracardiac artery and the coronary circulation. Circulation. 1990; 82:1449-66.

18. Beck CS, Leighninger DS. Scientific basis of the surgical treatment of coronary artery disease. JAMA. 1954;159:1264-71.

19. Shrager JB, Wain JC, Wright CD, Donahue DM, Vlahakes GJ, Moncure AC, et al. Omentum is highly effective in the management of complex cardiothoracic surgical problems. J Thorac Cardiovasc Surg. 2003; 125:526-32.

20. Tomanek RJ, Zheng W, Yue X. Growth factor activation in myocardial vascularization: therapeutic implications. Mol Cell Biochem. 2004;264:3-11.

21. Zhang QX, Magovern CJ, Mack CA, Budenbender KT, Ko W, Rosengart TK. Vascular endothelial growth factor is the major angiogenic factor in omentum: mechanism of the omentum-mediated angiogenesis. J Surg Res. 1997;67:147-54.

22. Sako A, Kitayama J, Yamaguchi H, Kaisaki S, Suzuki H, Fukatsu K, et al. Vascular endothelial growth factor synthesis by human omental mesothelial cells is augmented by fibroblast growth factor-2: possible role of mesothelial cell on the development of peritoneal metastasis. J Surg Res. 2003;115:113-20.

23. Ruel MA, Sellke FW, Bianchi C, Khan TA, Faro R, Zhang JP, et al Endogenous myocardial angiogenesis and revascularization using a gastric submucosal patch. Ann Thorac Surg. 2003;75:1443-9.

24. Kanamori T, Watanabe G, Yasuda T, Nagamine H, Kamiya H, Koshida Y. Hybrid surgical angiogenesis: omentopexy can enhance myocardial angiogenesis induced by cell therapy. Ann Thorac Surg. 2006; 81:160-7.

25. Ruel M, Wu GF, Khan TA, Voisine P, Bianchi C, Li J, et al. Inhibition of the cardiac angiogenic response to surgical FGF-2 therapy in a swine endothelial dysfunction model. Circulation. 2003;108:335-40.

26. Simons M. Angiogenesis, arteriogenesis, and diabetes: paradigm reassessed? J Am Coll Cardiol. 2005;46:835-7.

\section{Discussion}

Dr Todd K. Rosengart (Evanston, Ill). In this study the authors have convincingly demonstrated physiologically relevant increases in blood flow using an omental flap containing the GEA supplemented by bFGF application in the area of the omental flap. This work extends an increasing line of research looking at arteriogen- 
esis, as opposed to angiogenic techniques, which we're actually going to talk about in a minute.

I have 3 questions for you.

It is widely known that Vineberg-type procedures, be with it the internal thoracic artery, as has been described for several decades, or as now described, will increase myocardial blood flow. Do you have any data with the GEA control, that is, without using bFGF, to describe the relative contribution of the GEA alone as opposed to your bFGF angiogenic or arteriogenic therapies?

Second, we have previously demonstrated that the omentum is an extremely rich supply of vascular endothelial growth factor; in fact, it is the highest concentrations in the body. This presumably accounts for its role in abdominal healing, for example, and the use of omental flaps in general. Do you have any data looking at vascular endothelial growth factor alone as opposed to the omentum as your angiogenic/arteriogenic supplement?

Finally, similar work in this regard has been performed by Cohn and his associates, and has been reported previously, using essentially a very similar model, GEA as a Vineberg-type proce- dure. Do you have any information or are you aware of Dr Cohn's work compared with your own? Potentially that would provide some insights into the relative contribution of the GEA.

Dr Takaba. To answer your first question, we have investigated just omentopexy alone in a previous study of an acute myocardial infarction model. However, the effect of just omentopexy was lower than angiogenic factor alone. So now we can demonstrate just omentopexy.

Concerning your second question, basically we investigated bFGF, and bFGF is investigated for the effect of this. We have data for this.

Please repeat the third question.

Dr Rosengart. Are you familiar with Dr Cohn's prior work with a similar model in this area?

Dr Sellke. He used a gastric patch, based on the GEA, and did the same thing without the growth factor, but he found that there was increased perfusion in the chronically ischemic territory. Are you familiar with that?

Dr Takaba. I am not. Sorry. 
TABLE E1. Cine MRI analysis of global LV function

\begin{tabular}{lccc}
\hline & Group FG & Group F & Group N \\
\hline $\begin{array}{l}\text { Circumferential length at } \\
\text { end-diastole (mm) }\end{array}$ & $45.3 \pm 3.1^{*}$ & $44.4 \pm 2.8^{*}$ & $54.4 \pm 4.8$ \\
& & & \\
$\begin{array}{l}\text { Circumferential length at } \\
\text { end-systole (mm) }\end{array}$ & $30.3 \pm 3.8^{*} \dagger$ & $37.5 \pm 2.1^{*}$ & $43.4 \pm 2.5$ \\
& & & \\
LVEDV $\left(\mathrm{mm}^{3}\right)$ & $3410 \pm 345^{*}$ & $3253 \pm 411^{*}$ & $4354 \pm 508$ \\
LVESV $\left(\mathrm{mm}^{3}\right)$ & $1398 \pm 257^{*} \dagger$ & $2182 \pm 280^{*}$ & $3125 \pm 487$ \\
EF (\%) & $56.0 \pm 8.8^{*} \dagger$ & $30.8 \pm 7.8^{*}$ & $25.5 \pm 6.8$
\end{tabular}

$M R I$, Magnetic resonance imaging; $L V$, left ventricular; $L V E D V$, left ventricular end-diastolic volume; LVESV, left ventricular end-systolic volume; $E F$, ejection fraction. $* P<.01$ versus group $\mathrm{N}$. $† P<.01$ versus group $\mathrm{F}$. 